\title{
A DECISION TREE MODEL BASED ON CLINICAL AND LABORATORY MANIFESTATIONS AS PREDICTOR OF KIDNEY BIOPSY FINDINGS IN PATIENTS WITH LUPUS NEPHRITIS
}

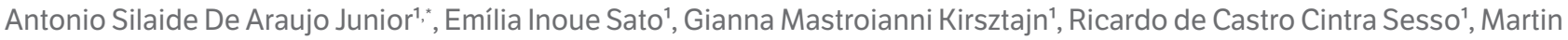
Fabio Jennings Simoes ${ }^{1}$, Alexandre Wagner Silva de Souza', Edgard Torres Dos Reis Neto'

1. Universidade Federal de São Paulo, São Paulo (SP), Brazil.

*Corresponding author: juniorantoniosilaide@gmail.com

\section{BACKGROUND}

Systemic lupus erythematosus (SLE) is a multisystemic autoimmune disease and lupus nephritis (LN) is one of the main causes of morbidity and mortality in these patients. Kidney biopsy is considered the gold standard for its diagnosis and is useful for guiding therapy. On the other hand, clinical and laboratory manifestations may be related to some subtypes of histopathological classes. The objectives of this work were to evaluate the correlation between clinical and laboratory data and as histological classes of LN and to develop an instrument that can assist in the identification of the histological class in LN.

\section{METHODOLOGY}

A retrospective study based on analysis of medical records of 80 SLE patients who were underwent kidney biopsy between 2010 and 2017. Clinical data such as age, race, hypertension, edema, and laboratory abnormalities such as serum creatinine, urinary sediment, 24-hour proteinuria, anti-dsDNA and serum complement were collected at the time of the kidney biopsy and correlated with renal biopsy outcome. Statistical analysis: association between two categorical variables was verified by Fischer's exact test. Means were compared by analysis of variance (ANOVA). A decision tree was construct via chi-square automatic interaction detector (CHAID) and logistic regression was performed. $p<0.05$ was considered significant.

\section{RESULTS}

The average age was $33 \pm 10.3$ years and the mean disease duration was $11.5 \pm 6.7$ years. The mean serum creatinine and 24 -hour proteinuria were, respectively, $1.85 \pm 1.88 \mathrm{mg} / \mathrm{dL}$ and $4.40 \pm 3.69 \mathrm{~g}$. Analyzing the laboratory variables as predictors of proliferative histological class (III or IV), we identified that those patients with abnormal urinary sediment had 13.96 times higher risk and those with anti-dsDNA antibodies positive had 4.96 times higher risk of presenting class III or IV ( $p<0.001)$. Our instrument presents a sensibility of $87.8 \%$ and specificity of $80 \%$ to detect class III or IV instead of class V using abnormal urinary sediment, anti-dsDNA antibodies and serum creatinine level as variables.

\section{CONCLUSIONS}

Patients with proliferative classes (III or IV) had more frequently hypertension, altered urinary sediment, higher creatinine levels, positive anti-dsDNA antibody, complement consumption and nephrotic pattern proteinuria. We propose a simple software as an instrument for histological class inference using clinical and laboratory variables with a good sensitivity and specificity that must be validated in another cohorts. 